\title{
Projective synchronization and parameter identification of a fractional-order chaotic system \\ KONG De-fu ${ }^{\mathrm{a}}$, ZHAO Xiao-shan ${ }^{\mathrm{b}^{*}}$
}

School of science, Tianjin University of Technology and Education , Tianjin 300222, China.

a775168682@qq.com, corresponding author Email:bxszhao678@126.com

Keyword: fractional-order chaotic system, projective synchronization, parameter identification.

\begin{abstract}
In this paper, projective synchronization and parameter identification of a fractional-order chaotic system is studied. Based on the fractional-order stability theory, a universal projective synchronization controller and parameter identification rules are designed and proved by using Lyapunov stability theory. Finally, the numerical simulations verify the correction of the method.
\end{abstract}

\section{Introduction}

As fractional calculus played an important role for the nonlinear dynamical systems, studying dynamics of fractional-order nonlinear system has become an interesting topic[1-5]. Since the pioneering work of Pecore and Carro introduced a method about synchronization between identical and non identical systems with different initial conditional, chaos synchronization had a great variety of applications in physics[6], ecological system[7], secure communications[8], etc. In this paper projective synchronization and parameter identification of a fractional-order chaotic system are investigated. A reasonable controller and parameter identification rules are designed and proved by Lyapunov stability theory. Numerical simulation coincide with the theoretical analysis.

\section{Problem Description.}

Consider the following chaotic drive and response systems:

$D_{t}^{q} x=F(x) \varphi+f(x)$

and

$$
D_{t}^{q} y=G(y) \psi+g(y)+U(x, y, \varphi, \psi)
$$

where $x, y \in R^{n}$ are the state vectors. $\varphi, \psi \in R^{m}$ are the parameter vector. $f(x)$ and $g(y)$ are the $n \times 1$ matrices. $F(x)$ and $G(y)$ are the $n \times m$ matrix. $U(x, y, \varphi, \psi)$ is the a suitable controller. Besides $0<q<1$.

Theorem 1. From the definition, the projective synchronization between the system (1) and (2) is achieved, if $\lim _{t \rightarrow \infty}\|e\|=\lim _{t \rightarrow \infty}\|y(t)-C x(t)\|=0$, where $C$ is called the scaling matrix. $C=\operatorname{diag}\left(\left[\alpha_{1}, \alpha_{2}, \ldots, \alpha_{n}\right]\right)$.

Theorem 2. Stability theorems of fractional-order system

Consider the following nonlinear system of fractional differential equation.

$$
\frac{d^{q} x}{d t^{q}}=A(x) x
$$

where $A(x) \in R^{n \times n}, 0<q<1, \quad x=\left(x_{1}, x_{2}, \ldots, x_{n}\right)^{\mathrm{T}}$ are state vectors. The fractional-order system (3) is asymptotically stable if and only if $\left|\arg \left(\lambda_{i}(A(x))\right)\right|>q \pi / 2, i=1,2, \ldots, \mathrm{n}$, where $\arg \left(\lambda_{i}(A(x))\right)$ denotes the argument of the eigenvalue $\lambda_{i}$ of $A$. 


\section{Projective Synchronization and Parameter Identification}

In this section, projective synchronization and parameter identification of Qi fractional-order chaotic system will be studied.

The drive system described through (1) is given by

$$
\left[\begin{array}{c}
\frac{d^{q} x_{1}}{d t^{q}} \\
\frac{d^{q} x_{2}}{d t^{q}} \\
\frac{d^{q} x_{3}}{d t^{q}}
\end{array}\right]=\left[\begin{array}{ccc}
x_{2}-x_{1} & 0 & 0 \\
0 & 0 & x_{1} \\
0 & -x_{3} & 0
\end{array}\right]\left[\begin{array}{l}
a \\
b \\
c
\end{array}\right]+\left[\begin{array}{c}
x_{2} x_{3} \\
-x_{2}-x_{1} x_{3} \\
x_{1} x_{2}
\end{array}\right]
$$

where $x_{1}, x_{2}, x_{3}$ are the state variables. The chaotic attractor of the system (4) for the order of derivative $q=0.915$ are displayed through Fig 1 for the parameters' values $a=35, b=8 / 3$, $c=80$.

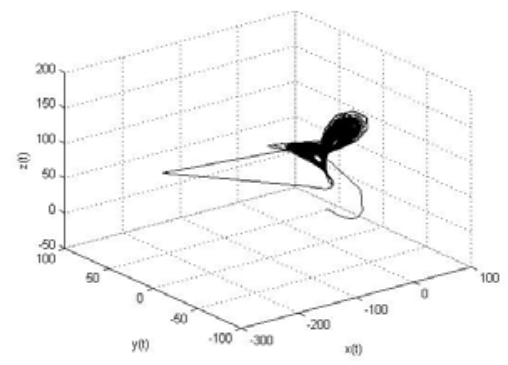

Fig.1 The phase portrait of the system (4) is shown in spaces $x-y-z$..

The response system described through (2) is given by

$$
\left[\begin{array}{c}
\frac{d^{q} y_{1}}{d t^{q}} \\
\frac{d^{q} y_{2}}{d t^{q}} \\
\frac{d^{q} y_{3}}{d t^{q}}
\end{array}\right]=\left[\begin{array}{ccc}
y_{2}-y_{1} & 0 & 0 \\
0 & 0 & y_{1} \\
0 & -y_{3} & 0
\end{array}\right]\left[\begin{array}{c}
\bar{a} \\
\bar{b} \\
\bar{c}
\end{array}\right]+\left[\begin{array}{c}
y_{2} y_{3} \\
-y_{2}-y_{1} y_{3} \\
y_{1} y_{2}
\end{array}\right]+\left[\begin{array}{l}
u_{1} \\
u_{2} \\
u_{3}
\end{array}\right]
$$

where $u_{1}, u_{2}, u_{3}$ are the control parameters.

In order to facilitate the following analysis, the error functions through theorem 1 are defined as $e_{1}=y_{1}-\alpha_{1} x_{1}, \quad e_{2}=y_{2}-\alpha_{2} x_{2}, \quad e_{3}=y_{3}-\alpha_{3} x_{3}$

And

$e_{a}=\bar{a}-a, e_{b}=\bar{b}-b, e_{c}=\bar{c}-c$

Now, we will choose suitable controllers and parameter identification rules to achieve projective synchronization according to theorem 2 .

From Eq.(1), (2), the error systems are obtained as

$D_{t}^{q} e=G(y) \psi+g(y)+U(x, y, \varphi, \psi)-C[F(x) \varphi+f(x)]$

Theorem 3. If the projective synchronization controllers are selected as $U=-g(y)+C f(x)+K e+D$

Where

$C=\left[\begin{array}{lll}\alpha & 0 & 0 \\ 0 & \alpha & 0 \\ 0 & 0 & \alpha\end{array}\right], K=\left[\begin{array}{ccc}0 & -a & 0 \\ -c & 0 & 0 \\ 0 & 0 & 0\end{array}\right], \quad D=\left[\begin{array}{c}0 \\ -y_{2}+\alpha x_{2} \\ 0\end{array}\right]$,

and identification laws of parameters are calculated as 


$$
\left\{\begin{array}{l}
\frac{d^{q} e_{a}}{d t^{q}}=-y_{2} e_{1}+y_{1} e_{1} \\
\frac{d^{q} e_{b}}{d t^{q}}=y_{3} e_{3} \\
\frac{d^{q} e_{c}}{d t^{q}}=-y_{1} e_{2}
\end{array}\right.
$$

then, the response system (5) is synchronized with the drive system (4) globally and asymptotically, i.e. $\lim _{t \rightarrow \infty}\|e(t)\|=0$.

proof: From Eq.(8), (9), the error systems are achieved as

$$
D_{t}^{q} e=G(y) \psi-C F(x) \varphi+K e+D
$$

A Lyapunov function is defined as follows:

$$
V=\frac{1}{2}\left(e_{1}^{2}+e_{2}^{2}+e_{3}^{2}+e_{a}^{2}+e_{b}^{2}+e_{c}^{2}\right)
$$

the time derivative of $V$ along the trajectory of the error system (11) leads to

$$
\begin{aligned}
& \frac{d^{q} V}{d t^{q}}=e_{1} \frac{d^{q} e_{1}}{d t^{q}}+e_{2} \frac{d^{q} e_{2}}{d t^{q}}+e_{3} \frac{d^{q} e_{3}}{d t^{q}}+e_{a} \frac{d^{q} e_{a}}{d t^{q}}+e_{b} \frac{d^{q} e_{b}}{d t^{q}}+e_{c} \frac{d^{q} e_{c}}{d t^{q}} \\
= & e_{1}\left(y_{2} e_{a}-y_{1} e_{a}-a e_{1}\right)+e_{2}\left(y_{1} e_{c}-e_{2}\right)+e_{3}\left(-y_{3} e_{b}-b e_{3}\right)+e_{a}\left(-y_{2} e_{1}+y_{1} e_{1}\right)+e_{b}\left(y_{3} e_{3}\right)+e_{c}\left(-y_{1} e_{2}\right) \\
= & -a e_{1}^{2}-e_{2}^{2}-b e_{3}^{2} \leq 0
\end{aligned}
$$

as $V \in R$ is positive definite function and $\frac{d^{q} V}{d t^{q}}$ is the negative definite function, so according to the Lyapunov stability theory, the response system (5) is synchronization to the drive system (4) asymptotically.

\section{Numerical Simulation and Results}

In this section, the initial conditions of the drive and response systems are $\left(x_{1}(0), x_{2}(0), x_{3}(0)\right)=(-1,-1,-2)$, and $\left(y_{1}(0), y_{2}(0), y_{3}(0)\right)=(-1,1,-5) \cdot \alpha=0.3$. The initial values of the estimated unknown parameter vectors of the systems are taken as $(\bar{a}, \bar{b}, \bar{c})=(20,-5,100)$. From figure 2 , it is seen the error vectors converge asymptotically to zero, and figure 3 show that the estimated parameter vectors converge to the original parameter vectors, respectively.
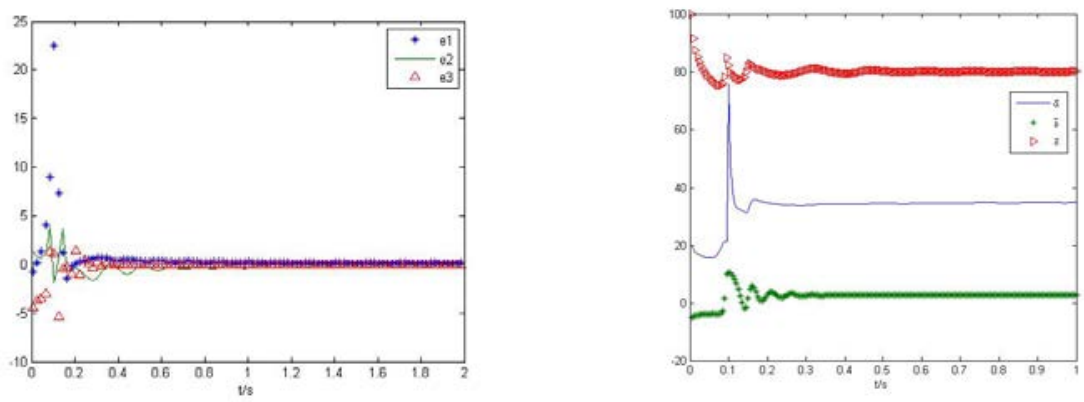

Fig.2 Trajectories of the errors function

Fig.3 Trajectories of the estimated parameter vectors

\section{Conclusion}

In this manuscript, projective synchronization and parameter identification of a fractional-order chaotic system is presented. Moreover, suitable projective synchronization controller and parameter identification rules are given by using the fractional-order stability and proved by Lyapunov stability theory. Finally, numerical simulations are performed to verify these results. 


\section{Reference}

[1] Hartley TT, Lorenzo CF, Qammer HK. Chaos in a fractional order Chua's system. IEEE Trans Circ Syst I 42 (1995) 485-490.

[2] Grigorenko I, Grigorenko E. Chaotic dynamics of the fractional Lorenz system. Phys Rev Lett 91 (2003) 034101

[3] Li CP. Peng GJ. Chaos in Chen’s system with a fractional order. Chaos Solitons Fract 22 (2004) 443-450.

[4] Li CG, Chen GR. Chaos and hyperchaos in the fractional-order Rösser equations. Physica A 341 (2004) 55-61.

[5] Wang XY. Wang MJ. Dynamic analysis of the fractional-order Liu system and its synchronization. Chaos 17 (2007) 033106.

[6] M. Lakshmanan, K. Murali, Chaos in Nonlinear Oscillator: Controlling and Synchronization, Word Scientific Pub Co Inc, 1996.

[7]. B. Blasius, A. Huppert, L, Stone. Complex dynamics and phase synchronization in spatially extended ecological system. Nature 399 (1999) 354-359.

[8] C.Y. Chee, D. Xu, Secure digital communication using controlled projective synchronization of chaos, Chaos solitons Fractal 23 (2005) 1063-1070. 